\title{
Some gamma function inequalities occurring in probability theory
}

\author{
Kwara Nantomah \\ Department of Mathematics, University for Development Studies, Navrongo Campus, \\ P. O. Box 24, Navrongo, UE/R, Ghana \\ E-mails: mykwarasoft@yahoo.com,knantomah@uds.edu.gh
}

Copyright (C)2015 Kwara Nantomah. This is an open access article distributed under the Creative Commons Attribution License, which permits unrestricted use, distribution, and reproduction in any medium, provided the original work is properly cited.

\begin{abstract}
By utilizing some basic analytical techniques, the authors establish some new Gamma function inequalities occurring in the study of Probability Theory.
\end{abstract}

Keywords: Gamma function, Completely monotonic function, Inequality.

2010 Mathematics Subject Classification: 33B15

\section{Introduction}

This paper is motivated by the inequality:

$\frac{(m+n) !}{(m+n)^{m+n}}<\frac{m ! n !}{m^{m} n^{n}}, \quad m, n \in Z^{+}$

which occures in Probability Theory. For instance, in a Bernoulli (or binomial) trial, there are two mutually exclusive outcomes, often referred to as "success" and "failure". If the probability of success is $p$, the probability of failure is $1-p$. The probability of $k$ succeesses in $n$ statistically independent trials, each with a probability of success $p$ is given by:

$P(k)=\left(\begin{array}{l}n \\ k\end{array}\right) p^{k}(1-p)^{n-k}$ where $\left(\begin{array}{l}n \\ k\end{array}\right)=\frac{n !}{k !(n-k) !}$

In particular, the probability of $m$ successes in $m+n$ trials with probability of success, $p$ on an individual trial is given by:

$P(m)=\left(\begin{array}{c}m+n \\ m\end{array}\right) p^{m}(1-p)^{n}<1$

for all $p \in(0,1)$ and $m, n \in Z^{+}$. That is;

$\frac{(m+n) !}{m ! n !} p^{m}(1-p)^{n}<1$. 
By setting $p=\frac{m}{m+n}$ in (2) the inequality (1) is obtained. This special inequality also appeared as Problem B-2 in the 65th William Lowell Putnam Mathematical Competition [3].

Let $\Gamma(x)$ and $\psi(x)$ respectively be the classical Gamma and Psi functions defined for $x \in R^{+}$by:

$\Gamma(x)=\lim _{n \rightarrow \infty}\left[\frac{n ! n^{x}}{x(x+1) \ldots(x+n)}\right]=\int_{0}^{\infty} t^{x-1} e^{-t} d t$,

$\psi(x)=\frac{d}{d x} \ln \Gamma(x)=\frac{\Gamma^{\prime}(x)}{\Gamma(x)}$.

It is well-known that the Gamma function satisfies the following properties.

$\Gamma(n+1)=n !, \quad n \in Z^{+}$,

$\Gamma(x+1)=x \Gamma(x), \quad x \in R^{+}$.

By utilizing the relation (3), inequality (1) takes the following elegant form:

$\frac{\Gamma(m+n+1)}{\Gamma(m+1) \Gamma(n+1)}<\frac{(m+n)^{m+n}}{m^{m} n^{n}}, \quad m, n \in Z^{+}$.

The purpose of this paper is to establish some related inequalities by using some basic analytical techniques. The results are presented in the following section.

\section{Main results}

Definition 2.1. Recall that a function $f:(0, \infty) \rightarrow R$ is said to be completely monotonic if it has derivatives of all orders and

$(-1)^{k} f^{(k)}(x) \geq 0 \quad$ for $\quad x \in(0, \infty), \quad k=0,1,2, \ldots$

For more information on completely monotonic functions, refer to [4], [5], [6] and the references therein.

Lemma 2.2. [2]. Suppose that $g:(0, \infty) \rightarrow(0,1]$ is a completely monotonic function. Then,

$\frac{g(x+y)}{g(x) g(y)} \geq 1 \quad$ for $\quad x, y \in R^{+} \cup\{0\}$.

Lemma 2.3. For $x, y \in R^{+}$, the following inequality holds true.

$\frac{\Gamma(x+y+1)}{\Gamma(x+1) \Gamma(y+1)} \leq \frac{(x+y)^{x+y}}{x^{x} y^{y}}$.

Proof. Let $g(x):=e^{x} x^{-x} \Gamma(x+1)$ for $x \in(0, \infty)$. Then, $\ln g(x)$ is completely monotonic on $(0, \infty)$. See [1] for instance. Consequently, $g$ is completely monotonic and the result follows directly from Lemma 2.2.

Remark 2.4. Observe from equation (7) that;

$\frac{x^{x} y^{y}}{(x+y)^{x+y}} \cdot \frac{\Gamma(x+y+1)}{\Gamma(x+1) \Gamma(y+1)} \leq 1$

Lemma 2.5. Let $S$ and $T$ be defined for $x \in\left[\frac{1}{2}, 1\right)$ and $y \in(0,1)$ by

$S(x)=e^{x} x^{-x} \Gamma(x+1) \quad$ and $\quad T(x, y)=\frac{S(x+y)}{S(x) S(y)}$.

Then,

(i) $S$ is increasing, and

(ii) $\frac{y^{y}}{e^{y}} \leq \frac{1}{S(y)} \leq T(x, y)$. 
Proof. (i) Let $f(x)=\ln S(x)$ for $x \in\left[\frac{1}{2}, 1\right)$. That is,

$f(x)=x-x \ln x+\ln \Gamma(x+1) . \quad$ Then,

$f(x)^{\prime}=-\ln x+\psi(x+1)>0 \quad$ for $\quad x \in\left[\frac{1}{2}, 1\right)$.

Hence $S(x)=e^{f(x)}$ is increasing. Notice that $\psi(x)>0$ for every $x \geq \frac{3}{2}$.

(ii) Proceed as follows for $x \in\left[\frac{1}{2}, 1\right)$ and $y \in(0,1)$ :

$T(x, y)=\frac{S(x+y)}{S(x) S(y)}=\frac{S(x+y)}{S(x)} \cdot \frac{1}{S(y)} \geq \frac{1}{S(y)}=\frac{y^{y}}{e^{y} \Gamma(y+1)} \geq \frac{y^{y}}{e^{y}}$

since $S(x)$ is increasing and $\Gamma(y+1)<1$ for $y \in(0,1)$.

Theorem 2.6. Let $x \in\left[\frac{1}{2}, 1\right)$ and $y \in(0,1)$. Then, the following inequalities hold true.

$\frac{(x+y)^{x+y}}{x^{x} e^{y}} \leq \frac{\Gamma(x+y+1)}{\Gamma(x+1) \Gamma(y+1)} \leq \frac{(x+y)^{x+y}}{x^{x} y^{y}}$

Proof. By equation (8) and Lemma 2.5, we obtain;

$\frac{y^{y}}{e^{y}} \leq T(x, y)=\frac{x^{x} y^{y}}{(x+y)^{x+y}} \cdot \frac{\Gamma(x+y+1)}{\Gamma(x+1) \Gamma(y+1)} \leq 1$

concluding the proof.

Lemma 2.7. Let $Q$ and $A$ be defined for $x \in\left[\frac{1}{2}, 1\right)$ and $y \in(0,1)$ by:

$Q(x)=\frac{\pi^{x} \Gamma(x+1)}{\gamma x^{x}} \quad$ and $\quad A(x, y)=\frac{Q(x+y)}{Q(x) Q(y)}$

where $\gamma=0.57721566490 \ldots$ is the Euler-Mascheroni's constant. Then,

(i) $Q$ is increasing, and

(ii) $\frac{\gamma y^{y}}{\pi^{y}} \leq \frac{1}{Q(y)} \leq A(x, y)$.

Proof. (i) Let $g(x)=\ln Q(x)$ for $x \in\left[\frac{1}{2}, 1\right)$. That is,

$g(x)=x \ln \pi+\ln \Gamma(x+1)-\ln \gamma-x \ln x . \quad$ Then,

$g(x)^{\prime}=\ln \pi-1+\psi(x+1)-\ln x \geq 0 \quad$ for $\quad x \in\left[\frac{1}{2}, 1\right)$.

Therefore, $Q(x)=e^{g(x)}$ is increasing.

(ii) Similarly, for $x \in\left[\frac{1}{2}, 1\right)$ and $y \in(0,1)$, we proceed as follows:

$A(x, y)=\frac{Q(x+y)}{Q(x) Q(y)}=\frac{Q(x+y)}{Q(x)} \cdot \frac{1}{Q(y)} \geq \frac{1}{Q(y)}=\frac{\gamma y^{y}}{\pi^{y} \Gamma(y+1)} \geq \frac{\gamma y^{y}}{\pi^{y}}$

since $Q(x)$ is increasing and $\Gamma(y+1)<1$ for $y \in(0,1)$.

Theorem 2.8. Assume that $x \in\left[\frac{1}{2}, 1\right)$ and $y \in(0,1)$. Then, the following inequalities are valid.

$\frac{(x+y)^{x+y}}{x^{x} \pi^{y}} \leq \frac{\Gamma(x+y+1)}{\Gamma(x+1) \Gamma(y+1)} \leq \frac{1}{\gamma} \cdot \frac{(x+y)^{x+y}}{x^{x} y^{y}}$ 
Proof. By equation (8) and Lemma 2.7, we obtain;

$\frac{\gamma y^{y}}{\pi^{y}} \leq A(x, y)=\frac{\gamma x^{x} y^{y}}{(x+y)^{x+y}} \cdot \frac{\Gamma(x+y+1)}{\Gamma(x+1) \Gamma(y+1)} \leq 1$

yielding the result.

Lemma 2.9. Let $U$ and $L$ be defined for $\alpha \geq 1, \beta \geq e, x \in\left[\frac{1}{2}, 1\right)$ and $y \in(0,1)$ by:

$U(x)=\frac{\alpha \beta^{x} \Gamma(x+1)}{x^{x}} \quad$ and $\quad L(x, y)=\frac{U(x+y)}{U(x) U(y)}$

where $e=2.71828 \ldots$ is the Euler's number. Then,

(i) $U$ is increasing, and

(ii) $\frac{y^{y}}{\alpha \beta^{y}} \leq \frac{1}{U(y)} \leq L(x, y)$.

Proof. (i) Let $h(x)=\ln U(x)$ for $\alpha \geq 1, \beta \geq e$ and $x \in\left[\frac{1}{2}, 1\right)$. That is,

$h(x)=\ln \alpha+x \ln \beta+\ln \Gamma(x+1)+x \ln x . \quad$ Then,

$h(x)^{\prime}=\ln \beta-1+\psi(x+1)-\ln x \geq 0$

Hence, $U(x)=e^{h(x)}$ is increasing.

(ii) Also, for $\alpha \geq 1, \beta \geq e$ and $x \in\left[\frac{1}{2}, 1\right)$, we have;

$L(x, y)=\frac{U(x+y)}{U(x) U(y)}=\frac{U(x+y)}{U(x)} \cdot \frac{1}{U(y)} \geq \frac{1}{U(y)}=\frac{y^{y}}{\alpha \beta^{y} \Gamma(y+1)} \geq \frac{y^{y}}{\alpha \beta^{y}}$

since $U(x)$ is increasing and $\Gamma(y+1)<1$ for $y \in(0,1)$.

Theorem 2.10. Suppose that $\alpha \geq 1, \beta \geq e, x \in\left[\frac{1}{2}, 1\right)$ and $y \in(0,1)$. Then, the following inequalities are valid.

$\frac{(x+y)^{x+y}}{x^{x} \beta^{y}} \leq \frac{\Gamma(x+y+1)}{\Gamma(x+1) \Gamma(y+1)} \leq \alpha \frac{(x+y)^{x+y}}{x^{x} y^{y}}$

Proof. By equation (8) and Lemma 2.9, we obtain;

$\frac{y^{y}}{\alpha \beta^{y}} \leq L(x, y)=\frac{1}{\alpha} \frac{x^{x} y^{y}}{(x+y)^{x+y}} \frac{\Gamma(x+y+1)}{\Gamma(x+1) \Gamma(y+1)} \leq 1$

establishing the result.

\section{Conclusion}

Some new two-sided inequalities related to the inequality (5) have been established. The procedure makes use of some basic analytical techniques.

\section{Acknowledgements}

The authors are very grateful to the anonymous refrees and the Editor for their useful comments and suggestions, which helped in the improvement of the quality of this paper. 


\section{References}

[1] CP. Chen and F. Qi, Logarithmically completely monotonic functions relating to the gamma function, J. Math. Anal. Appl, 321 (2006), 405-411.

[2] C. H. Kimberling, A Probabilistic Interpretation of Complete Monotonicity, Aequationes Math., 10 (1974), 152-164.

[3] 65th William Lowell Putnam Mathematical Competition: December 4, 2004, Available online at: http://kskedlaya.org/putnam-archive/2004.pdf, (Date Accessed: 01st Dec, 2014).

[4] V. Krasniqi, T. Mansour and A. Sh. Shabani, Some monotonicity properties and inequalities for $\Gamma$ and $\zeta$ functions, Mathematical Communications, 15(2) (2010), 365-376.

[5] M. Merkle, Completely Monotone Functions: A Digest, Analytic Number Theory, Approximation Theory, and Special Functions, (2014), 347-364.

[6] F. Qi and QM Luo, Complete monotonicity of a function involving the gamma function and applications, Periodica Mathematica Hungarica, 69(2) (2014), 159-169. 